\title{
Globular adiponectin enhances invasion in human breast cancer cells
}

\author{
EMILY FALK LIBBY ${ }^{1,2}$, JIANZHONG LIU ${ }^{2}$, YI LI $^{3}$, MONICA J. LEWIS ${ }^{2}$, \\ WENDY DEMARK-WAHNEFRIED ${ }^{1}$ and DOUGLAS R. HURST ${ }^{2}$
}

\begin{abstract}
Departments of ${ }^{1}$ Nutrition Sciences and ${ }^{2}$ Pathology, University of Alabama at Birmingham, Birmingham, AL 35294-0019, USA; ${ }^{3}$ State Key Laboratory of Oral Disease, West China Hospital of Stomatology, Sichuan University, Chengdu, Sichuan 610041, P.R. China
\end{abstract}

Received April 1,2015; Accepted November 5, 2015

DOI: $10.3892 / 01.2015 .3965$

\begin{abstract}
Every year, a large number of women succumb to metastatic breast cancer due to a lack of curative approaches for this disease. Adiponectin (AdipoQ) is the most abundant of the adipocyte-secreted adipokines. In recent years, there has been an interest in the use of AdipoQ and AdipoQ receptor agonists as therapeutic agents for the treatment of breast cancer. However, while multiple epidemiological studies have previously indicated that low levels of circulating plasma AdipoQ portend poor prognosis in patients with breast cancer, recent studies have reported that elevated expression levels of AdipoQ in breast tissue are correlated with advanced stages of the disease. Thus, the aim of the present study was to clarify the mechanism by which AdipoQ in breast tissue acts directly on tumor cells to regulate the early steps of breast cancer metastasis. In the present study, the effects of different AdipoQ isoforms on the metastatic potential of human breast cancer cells were investigated. The results revealed that globular adiponectin ( $\mathrm{gAd}$ ) promoted invasive cell morphology and significantly increased the migration and invasion abilities of breast cancer cells, whereas full-length adiponectin (fAd) had no effect on these cells. Additionally, gAd, but not fAd, increased the expression levels of microtubule-associated protein 1 light chain 3 beta (LC3B)-II and intracellular LC3B puncta, which are indicators of autophagosome formation, thus suggesting autophagic induction by gAd. Furthermore, the inhibition of autophagic function by autophagy-related
\end{abstract}

Correspondence to: Dr Douglas R. Hurst, Department of Pathology, University of Alabama at Birmingham, 1720 2nd Avenue South, Volker Hall G019A, Birmingham, AL 35294-0019, USA

E-mail: dhurst@uab.edu

Abbreviations: AdipoQ, adiponectin; fAd, full-length adiponectin; gAd, globular adiponectin; rapa, rapamycin; CQ, chloroquine; SFM, serum-free medium; NT, no treatment

Key words: adiponectin, autophagy, breast cancer, metastasis, invasion protein 7 knockdown attenuated the gAd-induced increase in invasiveness in breast cancer cells. Therefore, the results of the present study suggested that a specific AdipoQ isoform may enhance breast cancer invasion, possibly via autophagic induction. Understanding the roles of the different AdipoQ isoforms as microenvironmental regulatory molecules may aid the development of effective AdipoQ-based treatments for breast cancer.

\section{Introduction}

Molecules in the breast tumor microenvironment may impact the progression of breast tumor cells throughout all stages of the metastatic process (1). Adiponectin (AdipoQ) is a 244-amino acid protein, and the most abundant adipokine among the host of autocrine, endocrine and paracrine-acting adipokines secreted by adipocytes (2). Adipocytes are key constituents of the mammary stroma (3). Therefore, it is not surprising that the presence of AdipoQ has been previously reported in breast cancer and adjacent normal breast epithelial tissue (4-6), in addition to its well-characterized presence in circulating plasma, where its levels range from 2 to $30 \mu \mathrm{g} / \mathrm{ml}$ (2). Thus, we hypothesize that AdipoQ may act directly on tumor cells and potentially contribute to the microenvironmental regulation of the early steps of metastasis.

AdipoQ is synthesized as a $\sim 30 \mathrm{kDa}$ monomer, consisting of an amino-terminal sequence, a variable domain, a collagen-like domain and a carboxyl-terminal globular domain (2). This full-length monomer (fAd) assembles into higher-order structures such as trimers, hexamers and multimers of low, intermediate and high molecular weight $(7,8)$. AdipoQ additionally exists as a cleaved isoform named globular adiponectin (gAd), whereby fAd is truncated into a fragment primarily containing its globular domain $(9,10)$. This cleavage event is initiated by leukocyte elastase (10), a serine protease secreted by activated monocytes and neutrophils alongside breast cancer cells (11), which has been associated with poor prognosis in patients with breast cancer (11-13).

A substantial body of clinical and experimental evidence suggests that AdipoQ possesses an inhibitory function in the development (14-32) and progression $(22,24,32-35)$ of breast 
tumors. Notably, the majority of clinical evidence supporting antitumor roles for AdipoQ in breast cancer is based on circulating levels of serum AdipoQ, which often are not representative of tissue concentrations $(36,37)$. Furthermore, previous tissue-based studies have suggested an association between high expression levels of AdipoQ and cancer $(4,5)$. In addition, despite numerous observations suggesting that fAd and gAd diverge in biological function (9,38-40), the majority of studies on the role of AdipoQ in breast cancer have focused on fAd or total AdipoQ, without considering gAd (35). In previous studies, gAd has been observed to elicit pro-metastatic responses, including reduced hypoxia-associated apoptosis (41) and increased cell proliferation, migration, angiogenesis, activation of matrix metalloproteinases, secretion of proinflammatory cytokines and production of reactive oxygen species (ROS) (38-40).

Therefore, the present study aimed to assess the influence of gAd versus fAd on the metastatic potential of breast cancer cells. The results revealed a more invasive cell phenotype and an increase in indicators of autophagic induction upon addition of $\mathrm{gAd}$, but not fAd. In addition, the gAd-induced increase in invasion was observed to be partly dependent on autophagic function. An enhanced understanding of novel isoform-specific functions for AdipoQ in the breast tumor microenvironment may aid the development of novel therapeutic approaches for the treatment of metastatic breast cancer.

\section{Materials and methods}

Cells and cell culture. The metastatic human breast carcinoma cell line MDA-MB-231, previously described by Hurst et al (42), was acquired from the American Type Culture Collection (Manassas, VA, USA). The cell line was cultured in a 1:1 (v/v) mixture of Dulbecco's modified Eagle's medium and Ham's F-12 Nutrient Mixture (Thermo Fisher Scientific, Inc., Waltham, MA, USA) supplemented with $5 \%$ fetal bovine serum (FBS; Thermo Fisher Scientific, Inc.), 2 mM L-glutamine (Thermo Fisher Scientific, Inc.) and $0.02 \mathrm{mM}$ non-essential amino acids (Thermo Fisher Scientific, Inc.). Cells were maintained at $37^{\circ} \mathrm{C}$ with $5 \% \mathrm{CO}_{2}$ in a humidified atmosphere and were regularly tested for Mycoplasma spp. contamination with PlasmoTest $^{\mathrm{TM}}$ Reagent Kit (InvivoGen, San Diego, CA, USA), according to the manufacturer's protocol, and identified to be negative.

Transwell migration and invasion assays. Cell migration assays were performed with a Transwell chamber comprising 24 -well inserts with membranes of $8-\mu \mathrm{m}$ diameter pores (Corning Life Sciences, Manassas, VA, USA). Cells were seeded $\left(1 \times 10^{5}\right.$ cells/insert) in serum-free medium (SFM) and treated with $0.5 \mu \mathrm{g} / \mathrm{ml}$ human recombinant fAd (SouthernBiotech, Birmingham, AL, USA), $0.5 \mu \mathrm{g} / \mathrm{ml}$ human recombinant gAd (R\&D Systems, Inc., Minneapolis, MN, USA) or $100 \mathrm{nM}$ rapamycin (Sigma-Aldrich, St. Louis, MO, USA). The concentration of gAd used to treat the cells was selected based on a previous study reporting the relatively low levels of $\mathrm{gAd}$ compared with total AdipoQ (9). To ensure equal comparisons, the same concentration of fAd was used in the present study. Complete culture medium was added to the lower wells and employed as chemoattractant. Each treatment condition was evaluated in triplicate. Following incubation for $19 \mathrm{~h}$, non-migrated cells remaining on the upper surface of the insert membranes were removed using sterile cotton swabs (Thermo Fisher Scientific, Inc.), and the membranes were next washed with deionized water, fixed in $100 \%$ methanol (Thermo Fisher Scientific, Inc.) for $20 \mathrm{~min}$, washed and stained with crystal violet (Thermo Fisher Scientific, Inc.) for $18 \mathrm{~min}$. The inserts were air-dried overnight. Images of migrated cells ( 8 images/insert) were captured with ECLIPSE TE2000-U microscope (Nikon Corporation, Tokyo, Japan), and the average number of migrated cells per field was compared. Similar procedures were used for invasion assays with the following exceptions: Membranes were coated with a layer of Matrigel $^{\mathrm{TM}}$ Basement Membrane Matrix (BD Biosciences, San Jose, CA, USA), the seeding density was $5 \times 10^{5}$ cells/insert and the incubation period was $22 \mathrm{~h}$.

Three-dimensional (3D) cell morphology studies. 3D cell culture assays were performed in 24-well plates (Thermo Fisher Scientific, Inc.) with $400 \mu \mathrm{l} /$ well of Matrigel $^{\mathrm{TM}}$ Matrix Growth Factor Reduced (BD Biosciences). Cells were suspended in complete medium supplemented with $2 \%$ Matrige $^{\mathrm{TM}}$, plated at a density of $4 \times 10^{3}$ cells/well and incubated at $37^{\circ} \mathrm{C}$ for 9 days. A fresh layer of complete medium supplemented with Matrigel ${ }^{\mathrm{TM}}$ was added following 3 days of incubation, and 5 days later, the medium was replaced with SFM containing the following treatments: i) $0.5 \mu \mathrm{g} / \mathrm{ml}$ human recombinant fAd; ii) $0.5 \mu \mathrm{g} / \mathrm{ml}$ human recombinant gAd; or iii) $100 \mathrm{nM}$ rapamycin, individually or with $50 \mu \mathrm{M}$ chloroquine (Sigma-Aldrich). Each condition was evaluated in triplicate. Images were captured at 9 days post-incubation using ECLIPSE TE2000-U microscope.

Proliferation assay. Proliferation assays were performed as previously described (43). Briefly, cells were cultured in SFM, seeded onto a 96 -well tissue culture plate $\left(1 \times 10^{3}\right.$ cells/well; Thermo Fisher Scientific, Inc.) and treated with $0.5 \mu \mathrm{g} / \mathrm{ml}$ human recombinant fAd, $0.5 \mu \mathrm{g} / \mathrm{ml}$ human recombinant $\mathrm{gAd}$ or $100 \mathrm{nM}$ rapamycin. Cell viability was measured at 1, 3, 5 and 7 days post-incubation with alamarBlue ${ }^{\circledR}$ (Thermo Fisher Scientific, Inc.). Fluorescence intensity at 570/580 nm excitation/emission was determined via F-7000 Fluorescence Spectrophotometer (Hitachi, Ltd., Tokyo, Japan).

Immunoblot analysis. Cells grown in two-dimensional (2D) cultures were treated for 3 and $6 \mathrm{~h}$ under identical conditions to those used in 3D cell morphology studies. Whole-cell lysates were collected with $1 \mathrm{X}$ radioimmunoprecipitation assay lysis buffer (EMD Millipore, Billerica, MA, USA) containing $1 \mathrm{X}$ Halt $^{\mathrm{TM}}$ Protease and Phosphatase Inhibitor Single-Use Cocktail (Thermo Fisher Scientific, Inc.). Proteins were separated by sodium dodecyl sulfate-polyacrylamide gel electrophoresis (Bio-Rad Laboratories, Inc., Hercules, CA, USA) at $100 \mathrm{~V}$ for $2 \mathrm{~h}$ and transferred to nitrocellulose membranes (Bio-Rad Laboratories, Inc.). Membranes were next blocked for $1 \mathrm{~h}$ at room temperature using 5\% skimmed milk (Bio-Rad Laboratories, Inc.) dissolved in Tris-buffered saline (TBS) supplemented with $0.05 \%$ Tween 20 (TBST; Bio-Rad Laboratories, Inc.), and incubated overnight at $4{ }^{\circ} \mathrm{C}$ with the corresponding primary antibody. Membranes were 
then washed with TBST four times, incubated with the corresponding monoclonal donkey anti-rabbit (cat. no. NA934V) or sheep anti-mouse (cat. no. NA931V) IgG secondary antibody (1:10,000; GE Healthcare Life Sciences, Chalfont, $\mathrm{UK}$ ) for $1 \mathrm{~h}$ at room temperature and washed with TBST four times. Blots were developed using ECL Western Blotting Substrate (Thermo Fisher Scientific, Inc.) and Supersignal West Dura Extended Duration Substrate (Thermo Fisher Scientific, Inc.), and the results were quantified with Image Studio $^{\mathrm{TM}}$ Software version 4.0 (LI-COR Biotechnology, Lincoln, NE, USA). The following primary antibodies were employed: Rabbit anti-human polyclonal anti-microtubule-associated protein 1 light chain 3 beta (LC3B) $(1: 3,000$; cat. no. ab51520; Abcam, Cambridge, MA, USA), rabbit anti-human monoclonal autophagy related protein 7 (ATG7) (1:5,000; cat. no. 04-1055; EMD Millipore); and mouse anti-human monoclonal $\beta$-actin (1:10,000; cat. no. A5441; Sigma-Aldrich).

Immunofluorescence studies. Glass coverslips pretreated with $0.01 \%$ poly-L-lysine (Thermo Fisher Scientific, Inc.) were placed in 6-well culture plates, whereby cells were seeded and grown to $80-90 \%$ confluence. Next, cells were incubated with SFM containing $0.5 \mu \mathrm{g} / \mathrm{ml}$ human recombinant gAd or $100 \mathrm{nM}$ rapamycin, individually or with the addition of $50 \mu \mathrm{M}$ chloroquine. Each treatment condition was evaluated in triplicate. At 3 and $6 \mathrm{~h}$ post-incubation, cells were washed 4 times for $3 \mathrm{~min}$ in phosphate-buffered saline (PBS; (Thermo Fisher Scientific, Inc.), fixed in 3\% formaldehyde (Thermo Fisher Scientific, Inc.) dissolved in PBS for $45 \mathrm{~min}$ at room temperature, permeabilized with $0.5 \%$ Triton X-100 (Sigma-Aldrich) for $3 \mathrm{~min}$ at room temperature, blocked for $1 \mathrm{~h}$ with PBS containing $1 \%$ bovine serum albumin (BSA; Thermo Fisher Scientific, Inc.) and incubated overnight at $4^{\circ} \mathrm{C}$ with rabbit anti-human polyclonal LC3B antibody (1:2,000; cat. no. ab51520; Abcam) dissolved in PBS containing 1\% BSA. Cells were subsequently incubated with goat anti-rabbit IgG fluorescein isothiocyanate-labelled secondary antibody (1:2,000; cat. no. ab6717; Abcam) for $1 \mathrm{~h}$ at room temperature. The coverslips were then mounted onto glass slides (Thermo Fisher Scientific, Inc.) using VECTASHIELD ${ }^{\circledR}$ Antifade Mounting Medium with DAPI (catalogue no. H-1200; Vector Laboratories, Inc., Burlingame, CA, USA). Immunofluorescent images were captured with ECLIPSE TE2000-U fluorescence microscope.

RNA interference. Gene silencing assays were performed using small interfering (si)RNA targeting three non-overlapping sequences of ATG7 (MISSION ${ }^{\circledR}$ siRNAs catalogue nos.SASI_Hs01_00077648,SASI_Hs01_00077650 and SASI_Hs01_00077652, which were termed ATG7 siRNA1, 2 and 3, respectively; Sigma-Aldrich). MISSION ${ }^{\circledR}$ siRNA Universal Negative Control \#1 (Sigma-Aldrich) was selected as the non-targeting siRNA control, and referred to as scramble siRNA. To achieve transfection, Lipofectamine ${ }^{\circledR} 3000$ (Thermo Fisher Scientific, Inc.) was utilized according to the manufacturer's protocol.

Statistical analysis. Between-group differences were assessed by unpaired Student's t-test, and $\mathrm{P}<0.05$ was considered to indicate a statistically significant difference. Statistical analysis was performed with SigmaStat ${ }^{\circledR}$ software version 3.5 (Systat Software, Inc., Chicago, IL, USA).

\section{Results}

gAd increases the metastatic potential of breast cancer cells. The ability to migrate and invade through breast tissue stroma is an essential property of metastatic tumor cells (44). In order to investigate how different AdipoQ isoforms modulate this capacity, the effect of gAd and fAd on the 3D growth in Matrigel ${ }^{\mathrm{TM}}$ of the human metastatic breast carcinoma cell line 231 was evaluated. In the absence of serum, parental 231 cells grew with a relatively non-invasive grape-like morphology (Fig. 1A). By contrast, 231 cells treated with gAd developed spicules typical of a more invasive cell phenotype, whereas fAd-treated cells maintained a non-invasive grape-like structure similar to that of the untreated controls (Fig. 1A). Since none of the AdipoQ isoforms tested altered the proliferation ability of 231 cells in 2D culture (Fig. 1B), Transwell assays with and without Matrigel ${ }^{\mathrm{TM}}$ were subsequently performed to quantify the invasion and migration abilities of 231 cells. Treatment with gAd promoted migration by $221 \%$ (Fig. $1 \mathrm{C}$ and $\mathrm{D} ; \mathrm{P}<0.001$ ) and invasion by $91 \%$ (Fig. 1E and F; $\mathrm{P}<0.001$ ), compared with untreated cells. No significant alterations were noted following treatment with fAd. These results suggested that the different AdipoQ isoforms performed different functions. Thus, gAd appeared to promote the metastatic potential of 231 cells, contrarily to fAd.

Signaling pathways support the association between gAd and the induction of autophagy, a cellular stress response broadly considered to suppress tumorigenesis but promote the progression of established tumor cells (45). The authors of the present study have previously proposed a model in which autophagy is a plausible mechanism for gAd-stimulated breast cancer metastasis (35). In agreement with that model, the present study observed that gAd altered the metastatic potential of 231 cells in a manner akin to rapamycin, an established inducer of autophagy (46). Similar to gAd, rapamycin significantly increased cell migration (286\%; $\mathrm{P}=0.001$; Fig. 1C and D) and invasion (210\%; $\mathrm{P}<0.001$; Fig. 1E and F), and promoted the development of extended spikes in 3D cultures of 231 cells (Fig. 1A).

gAd promotes autophagic induction in breast cancer cells. Since gAd and rapamycin elicited similar invasive phenotypes in breast cancer cells, the association between different AdipoQ isoforms and autophagic induction was further investigated using rapamycin as positive control. Pharmacological inhibition of autophagy with chloroquine markedly reduced the gAd and rapamycin-induced increase in invasive morphology, whereas no clear alteration was observed in fAd-treated cells (Fig. 2A). Subsequent biochemical assays of autophagic induction corroborated these findings. LC3B is a widely used marker of autophagic induction, and the conversion of LC3B-I to LC3B-II, along with an increase in LC3B puncta, are considered to be indicative of autophagosome formation (46). To ensure an accurate comparison between treatments, any cellular alterations that occurred in 
A

NT

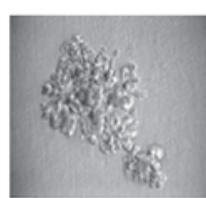

fAd

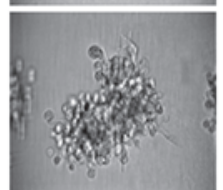

gAd

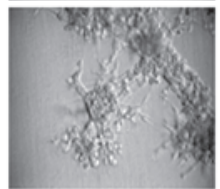

Rapa

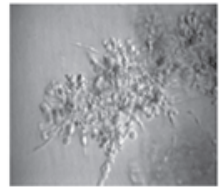

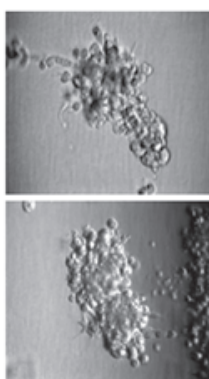
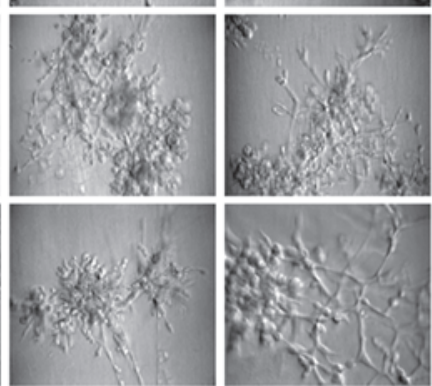

C

NT

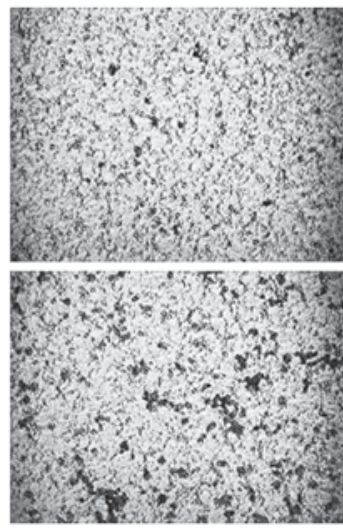

gAd

$\mathbf{E}$
NT

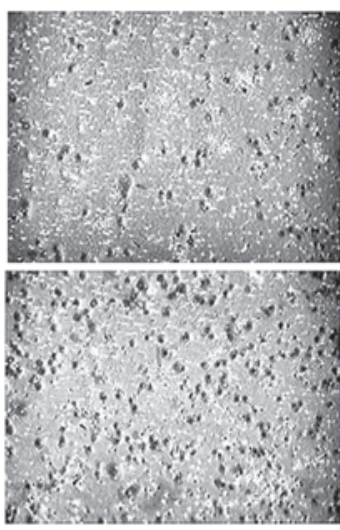

gAd

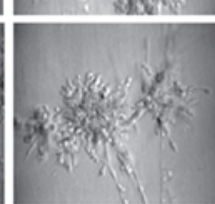

fAd

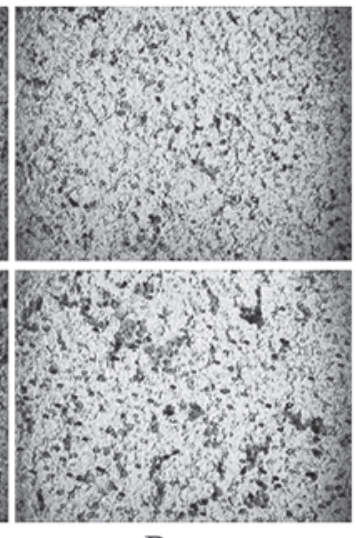

Rapa
B

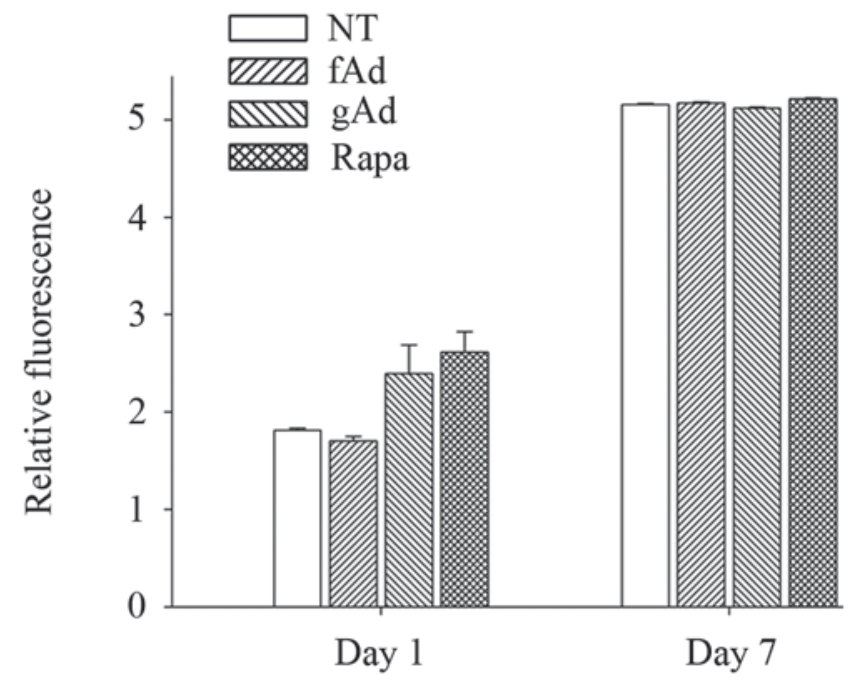

D

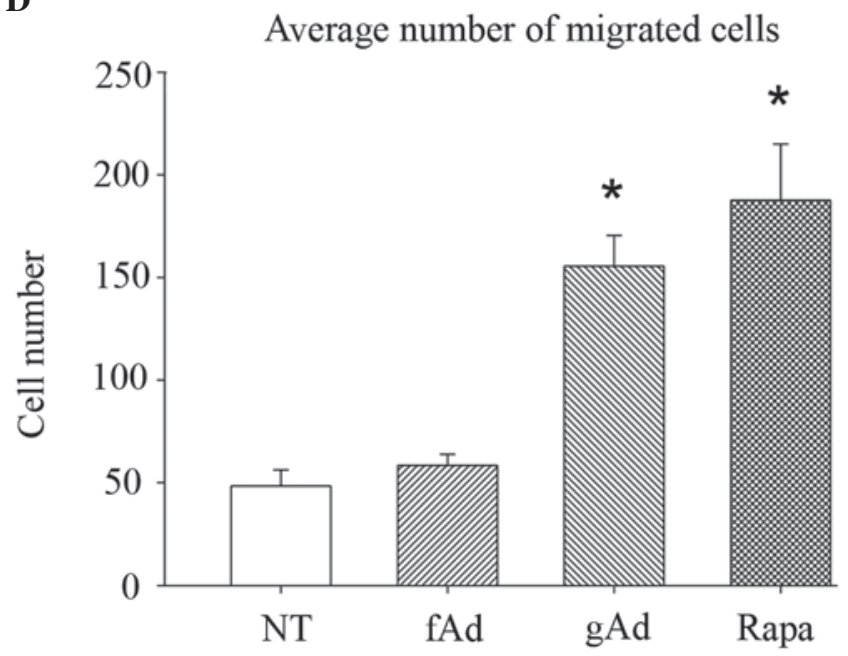

F

Average number of invaded cells

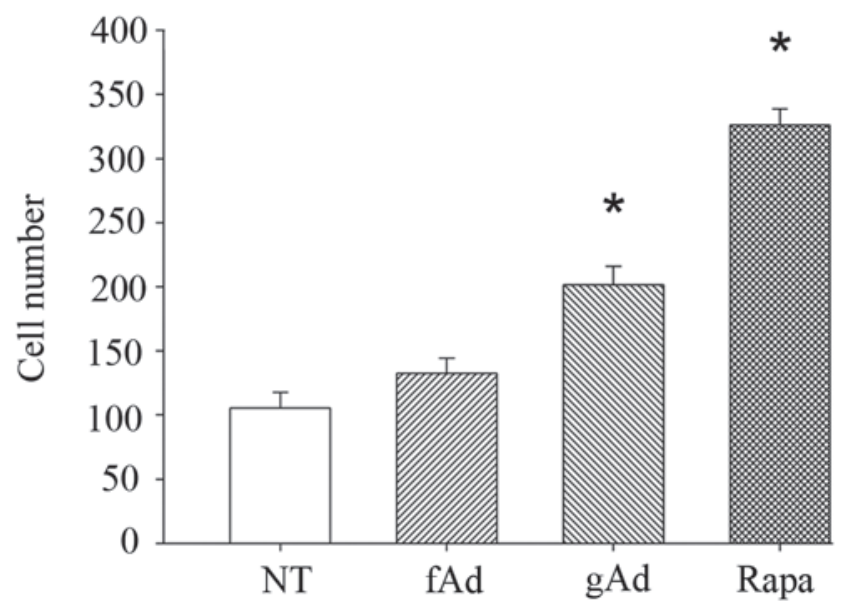

Figure 1. gAd increases the metastatic potential of breast cancer cells. (A) MDA-MB-231 cells were cultured in SFM, seeded onto Matrigel ${ }^{\mathrm{TM}}$-coated cell culture plates and treated with $0.5 \mu \mathrm{g} / \mathrm{ml}$ human recombinant fAd, $0.5 \mu \mathrm{g} / \mathrm{ml}$ human recombinant gAd or $100 \mathrm{nM}$ rapa. Untreated cells were used as negative controls. All conditions were evaluated in triplicate. Representative images of each replicate were obtained 9 days subsequent to seeding. (B) Cell proliferation was assessed with alamarBlue ${ }^{\circledR}$, which revealed that neither fAd, gAd nor rapa significantly altered cell growth over a 7-day period, compared with NT control cells. Each bar represents the mean fluorescence intensity obtained for each of the conditions, which were evaluated in quadruplicate. (C-F) Cells were cultured in SFM, seeded onto the top chamber of Transwell (C and D) migration and ( $\mathrm{E}$ and F) invasion plates, and treated with fAd ( $0.5 \mu \mathrm{g} / \mathrm{ml})$, gAd ( $0.5 \mu \mathrm{g} / \mathrm{ml})$ or rapa $(100 \mathrm{nM})$. Migration and invasion towards complete serum-containing medium were measured at 19 and $22 \mathrm{~h}$ post-incubation, respectively. $\mathrm{gAd}$ and rapa significantly increased cell migration and invasion, compared with NT controls. Data are represented as the mean \pm standard error from assays performed in triplicate. ${ }^{*} \leq 0.001$. SFM, serum-free medium; fAd, full-length adiponectin; gAd, globular adiponectin; rapa, rapamycin; NT, no treatment. 
A
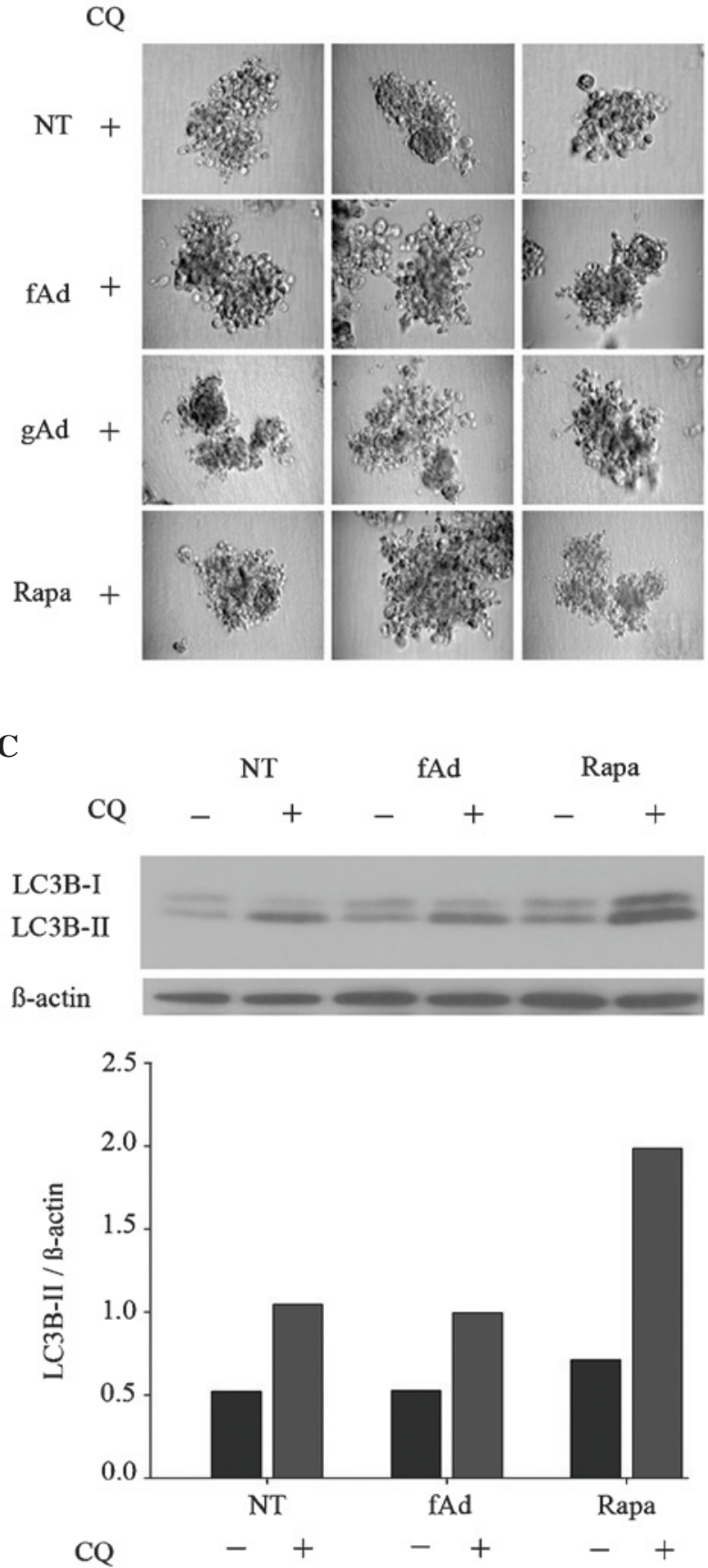

B

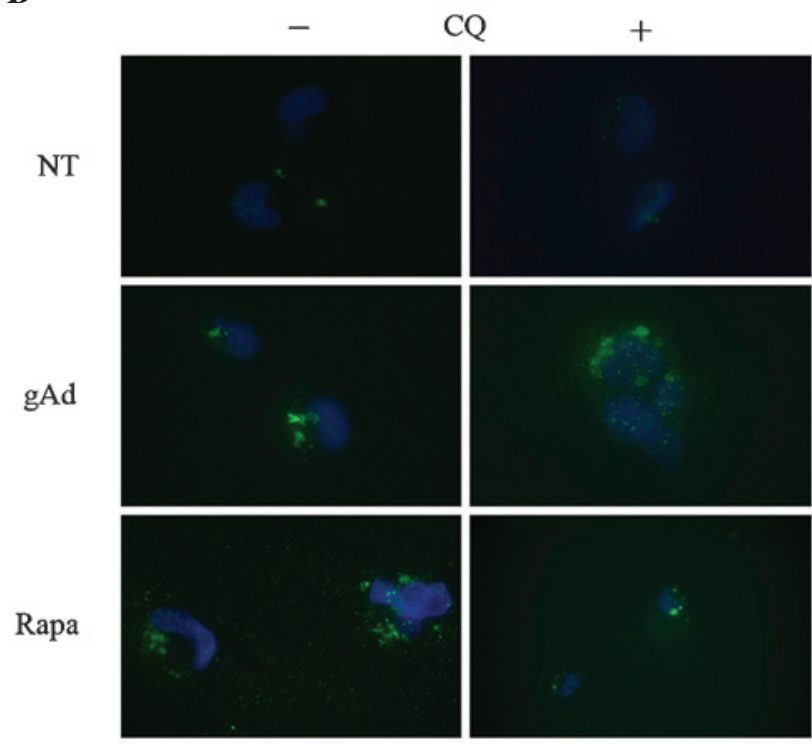

D
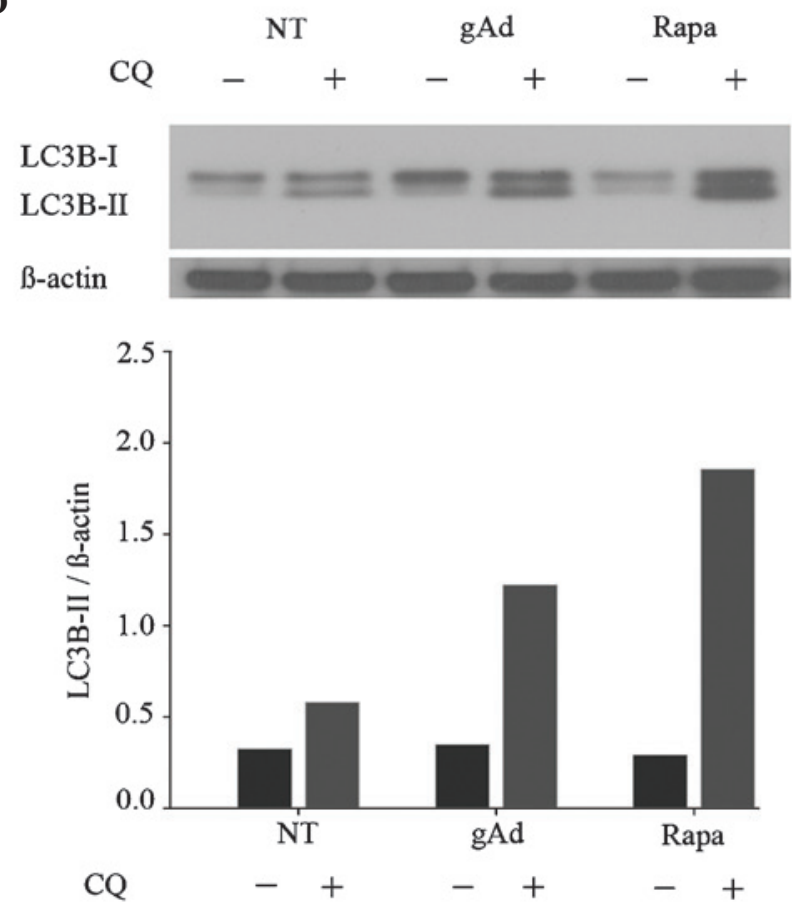

Figure 2.gAd promotes autophagic induction in breast cancer cells. (A) MDA-MB-231 cells cultured in serum-free medium were seeded onto Matrigel ${ }^{\mathrm{TM}}$-coated cell culture plates and treated with human recombinant fAd $(0.5 \mu \mathrm{g} / \mathrm{ml})$, human recombinant $\mathrm{gAd}(0.5 \mu \mathrm{g} / \mathrm{ml})$ or rapa (100 nM). Untreated cells were used as controls. CQ $(50 \mu \mathrm{M})$ was added to all the treatment conditions, which were evaluated in triplicate. Representative images of each replicate were obtained 9 days subsequent to seeding. (B) Immunofluorescence analysis revealed that gAd $(0.5 \mu \mathrm{g} / \mathrm{ml})$ and rapa $(100 \mathrm{nM})$ similarly increased the number of LC3B puncta. Each condition was evaluated in triplicate, and representative images are shown. (C and D) Immunoblotting results revealed that fAd (0.5 $\mu \mathrm{g} / \mathrm{ml})$ reduced, while gAd $(0.5 \mu \mathrm{g} / \mathrm{ml})$ and rapa $(100 \mathrm{nM})$ increased the expression levels of LC3B-II, compared with NT controls. CQ was added as an autophagy inhibitor to standardize the comparisons between the different conditions tested. gAd, globular adiponectin; fAd, full-length adiponectin; rapa, rapamycin; $\mathrm{CQ}$, chloroquine; LC3B, microtubule-associated protein 1 light chain 3 beta; NT, no treatment.

the presence of chloroquine were analyzed (46). Immunofluorescence analysis revealed that $\mathrm{gAd}$ and rapamycin similarly increased the number of LC3B puncta (Fig. 2B) Additionally, treatment with gAd and rapamycin increased the conversion of LC3B-I to LC3B-II in 231 cells by 110 and 220\%, respectively, compared with no treatment (Fig. 2C and D). In contrast, the levels of LC3B-II remained almost constant upon treatment with fAd. Therefore, the results of the present study suggested that gAd and fAd exerted disparate effects on autophagic induction.

Autophagic induction contributes to gAd-enhanced invasion. To assess whether autophagic induction may mediate the gAd-induced increase in migration and invasion observed in 231 cells, RNA interference was used to silence ATG7 in these cells. ATG7 is responsible for the conversion of LC3B-I 
A

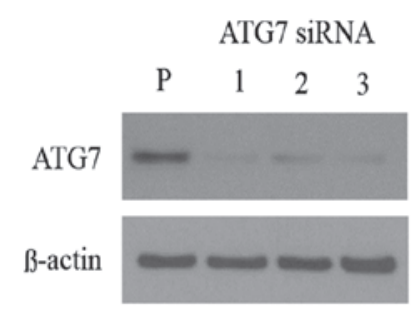

B

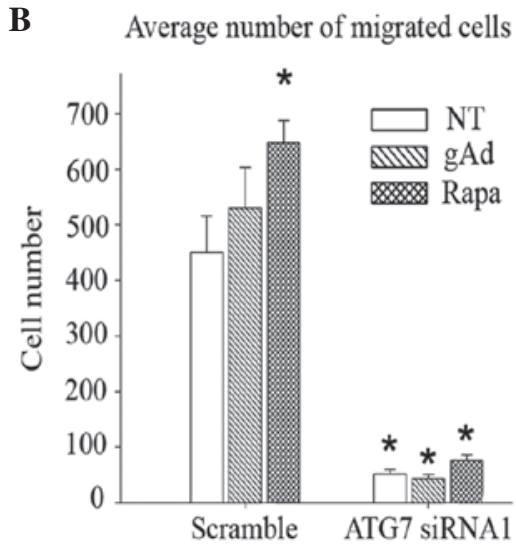

C Average number of invaded cells

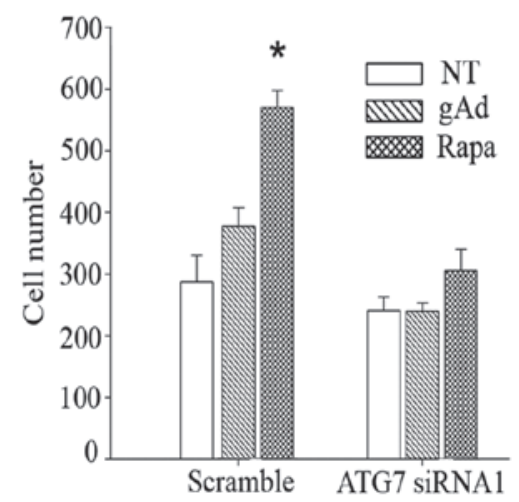

Figure 3. Autophagic induction contributes to gAd-enhanced invasion. (A) siRNA silencing of ATG7 was confirmed by immunoblot analysis. In cells where ATG7 had been knocked down, the enhanced (B) migration and (C) invasion abilities induced by gAd ( $0.5 \mu \mathrm{g} / \mathrm{ml})$ and rapa (100 $\mathrm{nM})$, respectively, were reduced, compared with cells transfected with scramble siRNA, which was used as non-targeting siRNA control. Data are represented as the mean \pm standard error of triplicate wells. "P<0.001. gAd, globular adiponectin; siRNA, small interfering RNA; ATG7, autophagy related protein 7; rapa, rapamycin; P, parental; NT, no treatment.

to LC3B-II and for the conjugation of ATG5 to ATG12, which is required for autophagosome formation during the initial stages of autophagy (46). Cells were transfected with three distinct siRNAs that targeted non-overlapping sequences of ATG7, in addition to a non-targeting negative control siRNA. Upon verifying the silencing efficiency of the different siRNAs by immunoblotting (Fig. 3A), an effective sequence, ATG7 siRNA1, was selected for the subsequent experimental assays. Silencing ATG7 attenuated the enhancement in cell migration caused by gAd and rapamycin (Fig. 3B), although knocking down ATG7 also significantly reduced migration in untreated cells $(\mathrm{P}<0.001)$. While gAd increased invasion by $32 \%(\mathrm{P}=0.09)$ in control cells (Fig. 3C), its effect was almost completely abolished $(<0.1 \% ; \mathrm{P}=0.97)$ in cells with impaired autophagic function. In addition, the rapamycin-induced increase in invasion was attenuated in ATG7 knockdown (28\%; $\mathrm{P}=0.14)$, compared with control (99\%; $\mathrm{P}<0.001)$ cells. Overall, the results of the present study are consistent with autophagic induction contributing to gAd-enhanced cell invasion.

\section{Discussion}

In the present study, the effects of different AdipoQ isoforms on the metastatic potential of breast cancer cells were compared. In the triple-negative human metastatic cell line MDA-MB-231, it was identified that gAd, but not fAd, increased migration, invasion and invasive cell morphology without altering proliferation. There is currently limited data on the roles of gAd in breast cancer $(20,31)$. Grossmann et al (20) reported that gAd reduced proliferation in 231 cells transfected with the estrogen receptor- $\alpha(E R \alpha)$ gene, but not in parental $E R \alpha-231$ cells (20). Mauro et al (31) observed that gAd inhibited proliferation in $\mathrm{ER} \alpha^{-} 231$ and SK-BR-3 cells, but increased proliferation in $\mathrm{ER} \alpha^{+}$ MCF7 and T47D cells. This group additionally reported that gAd downregulated the expression of cyclin D1 in 231 cells (47) and limited their anchorage-independent growth, while in MCF7 cells, gAd upregulated the expression of cyclin D1 (47) and increased their anchorage-independent growth, cell-cell adhesion and 3D growth. Furthermore, Jia et al (48) observed that an unspecified AdipoQ isoform increased the migration ability of MDA-MB-436 and MFM-223 cells. Although additional studies are required to fully elucidate the influence of gAd on breast cancer metastasis, the results of the present study suggest that gAd possesses unique functions compared with fAd regarding the promotion of cellular processes that enable the invasion and dissemination of metastatic breast tumor cells.

A number of studies on other types of cancer support the hypothesis that $\mathrm{gAd}$ and fAd act differentially in terms of eliciting metastasis-promoting cellular phenotypes $(38-41,49,50)$. Thus, gAd, but not fAd, increased migration and angiogenesis in human microvascular endothelial HMEC-1 cells (38). In the human colorectal adenocarcinoma cell line HT-29, gAd, but not fAd, increased the messenger RNA (mRNA) expression levels and the secretion of proinflammatory interleukin 8 (IL-8), granulocyte-macrophage colony-stimulating factor and monocyte chemoattractant protein-1, and promoted the nuclear translocation of nuclear factor-kappa B (NF-кB) (40). In human esophageal adenocarcinoma OE19 cells, gAd increased, while fAd reduced the activation of $N F-\kappa B$, the production of intracellular ROS and the mRNA levels of tumor necrosis factor- $\alpha$, IL- 8 and IL- 6 (49,50). In addition, gAd enhanced the production of ROS in monocytes and neutrophils treated with N-formyl-methionyl-leucyl-phenyl-alanine (39), and reduced the apoptosis induced by hypoxia-reoxygenation treatment (41). These observations, together with the results of the present study, highlight the importance of assessing the functions of gAd and fAd.

Based on potential associations between the $5^{\prime}$ adenosine monophosphate-activated protein kinase (AMPK)-mediated signaling pathways and the observation in the present study that gAd and rapamycin similarly increased metastatic potential in breast cancer cells, it was hypothesized that gAd may promote invasiveness of breast cancer cells partly by inducing autophagy. Cells exposed to a dual treatment of gAd or rapamycin plus chloroquine, a positive control for autophagy induction, exhibited a markedly less invasive 3D morphology than those treated with gAd and rapamycin alone. However, these findings 
must be interpreted with caution, since the autophagy inhibitor chloroquine is capable of exerting a wide range of effects that are not specific to autophagy (46). Nevertheless, the results of the present study were supported by the observation that gAd and rapamycin similarly upregulated LC3B-II, a key marker of autophagosome formation in the early stages of autophagy (46).

The association between gAd and autophagic induction in breast cancer cells revealed in the present study is consistent with the results of previous studies on other types of cancer (51-53). However, to the best of our knowledge, the aforementioned association has not been studied in breast cancer to date. In the present study, fAd did not alter the levels of LC3B-II, in contrast to a previous study that identified increased autophagosome and autophagolysosome formation in 231 cells following fAd treatment (54). In that study, the authors treated cells cultured in FBS-supplemented medium, as opposed to SFM, and used substantially higher concentrations of fAd than the ones used in the present study (54). Therefore, differences in the experimental conditions may have contributed to the inconsistencies observed across the two studies, although they were not evaluated in detail, since they are beyond the scope of the present study.

Knockdown of ATG7 reduced the gAd and rapamycin-induced promotion of cell invasion, providing evidence that gAd may act, at least in part, through autophagic pathways to affect metastatic behavior. However, ATG7 knockdown also reduced the migration ability of untreated cells, suggesting that gAd may promote migration through alternative pathways. By contrast, there was no significant alteration of invasion between the untreated control and ATG7 knockdown cells. Although the reasons for the differences in migration and invasion observed with ATG7 knockdown remain to be elucidated, the results of the present study are consistent with autophagic induction as a potential mechanism contributing to the gAd-enhanced invasiveness of breast cancer cells.

AdipoQ has been previously detected in breast cancer and adjacent tissue (4-6), although the distribution of $\mathrm{gAd}$ versus fAd has not been investigated in these previous studies. The authors of the present study recently proposed a model in which gAd is locally elevated in tumor tissue, where it enhances the potential of tumor cells to metastasize by upregulating the autophagic response through AdipoQ receptor 1 (ADIPOR1)-mediated activation of AMPK (35). A previous report in colorectal cancer identified significantly increased protein expression levels of gAd in tumor tissue of patients with colorectal cancer, compared with adjacent normal mucosa tissue. By contrast, fAd displayed the opposite trend (55). Furthermore, increased expression levels of gAd have been previously correlated with increased mRNA expression levels of AMPK and ADIPOR1 (2).

In conclusion, although the results of the present study are limited to a single cell line, they nonetheless provide initial evidence that the different AdipoQ isoforms present in the breast tumor microenvironment may exert different effects. The present study has demonstrated that gAd is able to differentially act on breast cancer cells to promote processes that facilitate metastatic progression, and that autophagic induction may mediate this effect. Furthermore, the present study is timely, considering the current interest in the preclinical development of AdipoQ and AdipoQ receptor-based therapies for the treatment of breast cancer (56-58) and the clinical use of autophagy modulators $(59,60)$. Additional studies are required to understand the specific association between different AdipoQ isoforms and metastasis, in order to optimize the effects of emerging therapies.

\section{Acknowledgements}

The present study was partly funded by the American Cancer Society (Atlanta, GA, USA) (grant no. RSG-11-259-01-CSM), METAvivor Research and Support, Inc. (Annapolis, MD, USA) (grant no. awarded to DRH) and the Cancer Prevention and Control Training Program of the University of Alabama at Birmingham (Birmingham, AL, USA) (grant no. R25 CA047888 awarded to WDW and EFL).

\section{References}

1. Joyce JA and Pollard JW: Microenvironmental regulation of metastasis. Nat Rev Cancer 9: 239-252, 2009.

2. Dalamaga M, Diakopoulos KN and Mantzoros CS: The role of adiponectin in cancer: A review of current evidence. Endocr Rev 33: 547-594, 2012.

3. Tan J, Buache E, Chenard MP, Dali-Youcef N and Rio MC: Adipocyte is a non-trivial, dynamic partner of breast cancer cells. Int J Dev Biol 55: 851-859, 2011.

4. Jeong YJ, Bong JG, Park SH, Choi JH and Oh HK: Expression of leptin, leptin receptor, adiponectin, and adiponectin receptor in ductal carcinoma in situ and invasive breast cancer. J Breast Cancer 14: 96-103, 2011.

5. Karaduman M, Bilici A, Ozet A, Sengul A, Musabak U and Alomeroglu M: Tissue levels of adiponectin in breast cancer patients. Med Oncol 24: 361-366, 2007.

6. Morad V, Abrahamsson A and Dabrosin C: Estradiol affects extracellular leptin:adiponectin ratio in human breast tissue in vivo. J Clin Endocrinol Metab 99: 3460-3467, 2014.

7. Waki H, Yamauchi T, Kamon J, Ito Y, Uchida S, Kita S, Hara K, Hada Y, Vasseur F, Froguel P, et al: Impaired multimerization of human adiponectin mutants associated with diabetes. Molecular structure and multimer formation of adiponectin. J Biol Chem 278: 40352-40363, 2003.

8. Pajvani UB, Du X, Combs TP, Berg AH, Rajala MW, Schulthess T, Engel J, Brownlee M and Scherer PE: Structure-function studies of the adipocyte-secreted hormone Acrp30/adiponectin. Implications for metabolic regulation and bioactivity. J Biol Chem 278: 9073-9085, 2003.

9. Fruebis J, Tsao TS, Javorschi S, Ebbets-Reed D, Erickson MRS, Yen FT, Bihain BE and Lodish HF: Proteolytic cleavage product of $30-\mathrm{kDa}$ adipocyte complement-related protein increases fatty acid oxidation in muscle and causes weight loss in mice. Proc Natl Acad Sci USA 98: 2005-2010, 2001.

10. Waki H, Yamauchi T, Kamon J, Kita S, Ito Y, Hada Y, Uchida S, Tsuchida A, Takekawa S and Kadowaki T: Generation of globular fragment of adiponectin by leukocyte elastase secreted by monocytic cell line THP-1. Endocrinology 146: 790-796, 2005.

11. Yamashita JI, Ogawa M, Ikei S, Omachi H, Yamashita SI, Saishoji T, Nomura K and Sato H: Production of immunoreactive polymorphonuclear leucocyte elastase in human breast cancer cells: Possible role of polymorphonuclear leucocyte elastase in the progression of human breast cancer. Br J Cancer 69: 72-76, 1994.

12. Foekens JA, Ries C, Look MP, Gippner-Steppert C, Klijn JG and Jochum M: The prognostic value of polymorphonuclear leukocyte elastase in patients with primary breast cancer. Cancer Res 63: 337-341, 2003.

13. Akizuki M, Fukutomi T, Takasugi M, Takahashi S, Sato T, Harao M, Mizumoto T and Yamashita J: Prognostic significance of immunoreactive neutrophil elastase in human breast cancer: Long-term follow-up results in 313 patients. Neoplasia 9: 260-264, 2007.

14. Liu LY, Wang M, Ma ZB, Yu LX, Zhang Q, Gao DZ, Wang F and Yu ZG: The role of adiponectin in breast cancer: A meta-analysis. PLoS One 8: e73183, 2013. 
15. Körner A, Pazaitou-Panayiotou K, Kelesidis T, Kelesidis I, Williams CJ, Kaprara A, Bullen J, Neuwirth A, Tseleni S, Mitsiades N, et al: Total and high-molecular-weight adiponectin in breast cancer: In vitro and in vivo studies. J Clin Endocrinol Metab 92: 1041-1048, 2007.

16. Chen DC, Chung YF, Yeh YT, Chaung HC, Kuo FC, Fu OY, Chen HY, Hou MF and Yuan SS: Serum adiponectin and leptin levels in Taiwanese breast cancer patients. Cancer Lett 237: 109-114, 2006.

17. Mantzoros C, Petridou E, Dessypris N, Chavelas C, Dalamaga M, Alexe DM, Papadiamantis Y, Markopoulos C, Spanos E, Chrousos G and Trichopoulos D: Adiponectin and breast cancer risk. J Clin Endocrinol Metab 89: 1102-1107, 2004.

18. Miyoshi Y, Funahashi T, Kihara S, Taguchi T, Tamaki Y, Matsuzawa Y and Noguchi S: Association of serum adiponectin levels with breast cancer risk. Clin Cancer Res 9: 5699-5704, 2003.

19. Tworoger SS, Eliassen AH, Kelesidis T, Colditz GA, Willett WC, Mantzoros CS and Hankinson SE: Plasma adiponectin concentrations and risk of incident breast cancer. J Clin Endocrinol Metab 92: 1510-1516, 2007.

20. Grossmann ME, Nkhata KJ, Mizuno NK, Ray A and Cleary MP: Effects of adiponectin on breast cancer cell growth and signaling. Br J Cancer 98: 370-379, 2008.

21. Dos Santos E, Benaitreau D, Dieudonne MN, Leneveu MC, Serazin V, Giudicelli Y and Pecquery R: Adiponectin mediates an antiproliferative response in human MDA-MB 231 breast cancer cells. Oncol Rep 20: 971-977, 2008.

22. Duggan C, Irwin ML, Xiao L, Henderson KD, Smith AW, Baumgartner RN, Baumgartner KB, Bernstein L, Ballard-Barbash R and McTiernan A: Associations of insulin resistance and adiponectin with mortality in women with breast cancer. J Clin Oncol 29: 32-39, 2011.

23. Arditi JD, Venihaki M, Karalis KP and Chrousos GP Antiproliferative effect of adiponectin on MCF7 breast cancer cells: A potential hormonal link between obesity and cancer. Horm Metab Res 39: 9-13, 2007.

24. Taliaferro-Smith L, Nagalingam A, Zhong D, Zhou W, Saxena NK and Sharma D: LKB1 is required for adiponectin-mediated modulation of AMPK-S6K axis and inhibition of migration and invasion of breast cancer cells. Oncogene 28 : 2621-2633, 2009.

25. Taliaferro-Smith L, Nagalingam A, Knight BB, Oberlick E, Saxena NK and Sharma D: Integral role of PTP1B in adiponectin-mediated inhibition of oncogenic actions of leptin in breast carcinogenesis. Neoplasia 15: 23-38, 2013

26. Treeck O, Lattrich C, Juhasz-Boess I, Buchholz S, Pfeiler G and Ortmann O: Adiponectin differentially affects gene expression in human mammary epithelial and breast cancer cells. Br J Cancer 99: 1246-1250, 2008

27. Wang Y, Lam JB, Lam KS, Liu J, Lam MC, Hoo RL, Wu D, Cooper GJ and Xu A: Adiponectin modulates the glycogen synthase kinase-3beta/beta-catenin signaling pathway and attenuates mammary tumorigenesis of MDA-MB-231 cells in nude mice. Cancer Res 66: 11462-11470, 2006.

28. Dieudonne MN, Bussiere M, Dos Santos E, Leneveu MC, Giudicelli Y and Pecquery R: Adiponectin mediates antiproliferative and apoptotic responses in human MCF7 breast cancer cells. Biochem Biophys Res Commun 345: 271-279, 2006.

29. Nakayama S, Miyoshi Y, Ishihara H and Noguchi S: Growth-inhibitory effect of adiponectin via adiponectin receptor 1 on human breast cancer cells through inhibition of $\mathrm{S}$-phase entry without inducing apoptosis. Breast Cancer Res Treat 112: 405-410, 2008

30. Pfeiler GH, Buechler C, Neumeier M, Schäffler A, Schmitz G, Ortmann O and Treeck O: Adiponectin effects on human breast cancer cells are dependent on 17-beta estradiol. Oncol Rep 19: 787-793, 2008

31. Mauro L, Pellegrino M, De Amicis F, Ricchio E, Giordano F, Rizza P, Catalano S, Bonofiglio D, Sisci D, Panno ML and Andò S: Evidences that estrogen receptor $\alpha$ interferes with adiponectin effects on breast cancer cell growth. Cell Cycle 13 553-564, 2014

32. Kim KY, Baek A, Hwang JE, Choi YA, Jeong J, Lee MS, Cho DH, Lim JS, Kim KI and Yang Y: Adiponectin-activated AMPK stimulates dephosphorylation of AKT through protein phosphatase 2A activation. Cancer Res 69: 4018-4026, 2009.

33. Kang JH, Yu BY and Youn DS: Relationship of serum adiponectin and resistin levels with breast cancer risk. J Korean Med Sci 22: 117-121, 2007
34. Macis D, Gandini S, Guerrieri-Gonzaga A, Johansson H, Magni P, Ruscica M, Lazzeroni M, Serrano D, Cazzaniga M, Mora S, et al: Prognostic effect of circulating adiponectin in a randomized $2 \times 2$ trial of low-dose tamoxifen and fenretinide in premenopausal women at risk for breast cancer. J Clin Oncol 30: 151-157, 2012.

35. Libby EF, Frost AR, Demark-Wahnefried W and Hurst DR Linking adiponectin and autophagy in the regulation of breast cancer metastasis. J Mol Med Berl 92: 1015-1023, 2014.

36. Llanos AA, Dumitrescu RG, Marian C, Makambi KH, Spear SL, Kallakury BV, Perry DJ, Convit RJ, Platek ME, Millen AE, et al: Adipokines in plasma and breast tissues: Associations with breast cancer risk factors. Cancer Epidemiol Biomarkers Prev 21: 1745-1755, 2012

37. Sonmez B, Seker M, Bilici A, Yavuz ErkalF, Oven Ustaalioglu BB, Gumus M, Ozturk Guler D, Karaduman M, Gezen C, Eser M, et al: Is there any correlation among adiponectin levels in serum, tumor tissue and normal tissue of the same patients with breast cancer? J BUON 16: 227-232, 2011.

38. Adya R, Tan BK, Chen J and Randeva HS: Protective actions of globular and full-length adiponectin on human endothelial cells: Novel insights into adiponectin-induced angiogenesis. J Vasc Res 49: 534-543, 2012.

39. Chedid P, Hurtado-Nedelec M, Marion-Gaber B, Bournier O, Hayem G, Gougerot-Pocidalo MA, Frystyk J, Flyvbjerg A, El Benna J and Marie JC: Adiponectin and its globular fragment differentially modulate the oxidative burst of primary human phagocytes. Am J Pathol 180: 682-692, 2012.

40. Ogunwobi OO and Beales IL: Adiponectin stimulates proliferation and cytokine secretion in colonic epithelial cells. Regul Pept 134: 105-113, 2006.

41. Park M, Youn B, Zheng XL, Wu D, Xu A and Sweeney G: Globular adiponectin, acting via AdipoR1/APPL1, protects H9c2 cells from hypoxia/reoxygenation-induced apoptosis. PLoS One 6: e19143, 2011.

42. Hurst DR, Xie Y, Vaidya KS, Mehta A, Moore BP, Accavitti-Loper MA, Samant RS, Saxena R, Silveira AC and Welch DR: Alterations of BRMS1-ARID4A interaction modify gene expression but still suppress metastasis in human breast cancer cells. J Biol Chem 283: 7438-7444, 2008.

43. Cody JJ, Markert JM and Hurst DR: Histone deacetylase inhibitors improve the replication of oncolytic herpes simplex virus in breast cancer cells. PLoS One 9: e92919, 2014.

44. Talmadge JE and Fidler IJ: AACR centennial series: The biology of cancer metastasis: Historical perspective. Cancer Res 70: 5649-5669, 2010

45. White E: Deconvoluting the context-dependent role for autophagy in cancer. Nat Rev Cancer 12: 401-410, 2012.

46. Klionsky DJ, Abdalla FC, Abeliovich $\mathrm{H}$, Abraham RT, Acevedo-Arozena A, Adeli K, Agholme L, Agnello M, Agostinis P, Aguirre-Ghiso JA, et al: Guidelines for the use and interpretation of assays for monitoring autophagy. Autophagy 8: 445-544, 2012

47. Mauro L, Pellegrino M, Giordano F, Ricchio E, Rizza P, De Amicis F, Catalano S, Bonofiglio D, Panno ML and Andò S: Estrogen receptor- $\alpha$ drives adiponectin effects on cyclin D1 expression in breast cancer cells. FASEB J 29: 2150-2160, 2015.

48. Jia Z, Liu Y and Cui S: Adiponectin induces breast cancer cell migration and growth factor expression. Cell Biochem Biophys 70: 1239-1245, 2014

49. Zhang R, Wu J, Liu D, Shan $\mathrm{H}$ and Zhang J: Anti-inflammatory effect of full-length adiponectin and proinflammatory effect of globular adiponectin in esophageal adenocarcinoma cells. Oncol Res 21: 15-21, 2013.

50. Zhang R, Yin X, Shi H, Wu J, Shakya P, Liu D and Zhang J: Adiponectin modulates DCA-induced inflammation via the ROS/NF- $\kappa$ B signaling pathway in esophageal adenocarcinoma cells. Dig Dis Sci 59: 89-97, 2014.

51. Habeeb BS, Kitayama J and Nagawa H: Adiponectin supports cell survival in glucose deprivation through enhancement of autophagic response in colorectal cancer cells. Cancer Sci 102: 999-1006, 2011.

52. Nepal S, Kim MJ, Lee ES, Kim JA, Choi DY, Sohn DH, Lee SH, Song K, Kim SH, Jeong GS, et al: Modulation of Atg5 expression by globular adiponectin contributes to autophagy flux and suppression of ethanol-induced cell death in liver cells. Food Chem Toxicol 68: 11-22, 2014

53. Nepal S and Park PH: Activation of autophagy by globular adiponectin attenuates ethanol-induced apoptosis in HepG2 cells: Involvement of AMPK/FoxO3A axis. Biochim Biophys Acta 1833: 2111-2125, 2013. 
54. Liu J, Xu A, Lam KS, Wong NS, Chen J, Shepherd PR and Wang Y: Cholesterol-induced mammary tumorigenesis is enhanced by adiponectin deficiency: Role of LDL receptor upregulation. Oncotarget 4: 1804-1818, 2013.

55. Vetvik KK, Sonerud T, Lindeberg M, Lüders T, Størkson RH, Jonsdottir K, Frengen E, Pietiläinen KH and Bukholm I: Globular adiponectin and its downstream target genes are up-regulated locally in human colorectal tumors: Ex vivo and in vitro studies. Metabolism 63: 672-681, 2014.

56. Delort L, Jardé T, Dubois V, Vasson MP and Caldefie-Chézet F. New insights into anticarcinogenic properties of adiponectin: A potential therapeutic approach in breast cancer? Vitam Horm 90: 397-417, 2012.

57. Khan S, Shukla S, Sinha S and Meeran SM: Role of adipokines and cytokines in obesity-associated breast cancer: Therapeutic targets. Cytokine Growth Factor Rev 24: 503-513, 2013.
58. Otvos L Jr, Haspinger E, La Russa F, Maspero F, Graziano P, Kovalszky I, Lovas S, Nama K, Hoffmann R, Knappe D, et al: Design and development of a peptide-based adiponectin receptor agonist for cancer treatment. BMC Biotechnol 11: 90 , 2011.

59. Gewirtz DA: The four faces of autophagy: Implications for cancer therapy. Cancer Res 74: 647-651, 2014.

60. Vinayak S and Carlson RW: mTOR inhibitors in the treatment of breast cancer. Oncology (Williston Park) 27: 38-48, 2013. 